\title{
OSCIENCE CHINA
}

\section{Information Sciences}

\section{A Large-scale Multi-objective Flights Conflict Avoidance Approach Supporting 4D Trajectory Operation}

Guan Xiangmin

Citation: SCIENCE CHINA Information Sciences ; doi: 10.1007/s11432-016-9024-y

View online: http://engine.scichina.com/doi/10.1007/s11432-016-9024-y

Published by the Science China Press

\section{Articles you may be interested in}

A large-scale flight multi-objective assignment approach based on multi-island parallel evolution algorithm with cooperative coevolutionary

SCIENCE CHINA Information Sciences 59, 072201 (2016);

Multi-objective optimization of space station short-term mission planning

SCIENCE CHINA Technological Sciences 58, 2169 (2015);

Multi-level multi-area hybrid automatic voltage control system and its trial operation in Northeast China Grid SCIENCE CHINA Technological Sciences 54, 2501 (2011);

Synergy methodology for multi-objective operational control of reservoirs in Yellow River basin Science in China Series E-Technological Sciences 47, 212 (2004);

Multi-objective interplanetary trajectory optimization combining low-thrust propulsion and gravity-assist maneuvers SCIENCE CHINA Technological Sciences 55, 841 (2012); 


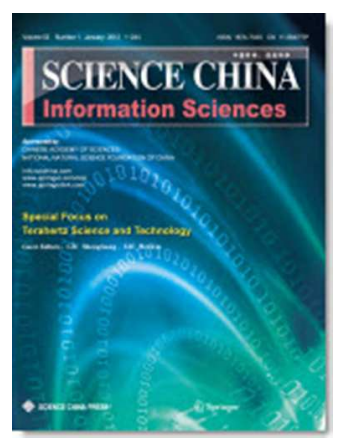

\section{A Large-scale Multi-objective Flights Conflict Avoidance Approach Supporting 4D Trajectory Operation}

\begin{tabular}{|r|l|}
\hline Journal: & SCIENCE CHINA Information Sciences \\
\hline Manuscript ID & SCIS-2016-0554.R2 \\
\hline Manuscript Type: & Research Paper ( $<15$ pages) \\
\hline $\begin{array}{r}\text { Date Submitted by the } \\
\text { Author: }\end{array}$ & 20-Jan-2017 \\
\hline Complete List of Authors: & $\begin{array}{l}\text { Guan, Xiangmin; Civil Aviation Management Institute of China, } \\
\text { Department of General Aviation }\end{array}$ \\
\hline Keywords: & $\begin{array}{l}\text { Air traffic management, Conflict avoidance, Combinatorial } \\
\text { optimization, Multi-objective, Cooperative co-evolution }\end{array}$ \\
\hline Speciality: & $\begin{array}{l}\text { Artificial Intelligence } \backslash \text { Pattern Recognition } \backslash \text { Database and } \\
\text { Knowledge-Based Systems }<\text { Computer Science \& Technology }\end{array}$ \\
\hline
\end{tabular}




\title{
A Large-scale Multi-objective Flights Conflict Avoidance Approach Sup-porting 4D Trajectory Operation
}

\author{
GUAN Xiangmin ${ }^{1}$, ZHANG Xuejun $^{2 *}$, LV Renli ${ }^{1}$, Jun Chen $^{3} \&$ Michal Weiszer $^{3}$ \\ ${ }^{1}$ Department of General Aviation, Civil Aviation Management Institute of China, Beijing 100102, PR China; \\ ${ }^{2}$ School of Electronic and Information Engineering, Beihang University, Beijing 100191, PR China; \\ ${ }^{3}$ School of Engineering, University of Lincoln, Lincoln, UK, LN6 7TS
}

\begin{abstract}
Recently, the long-term conflict avoidance approaches based on large-scale flights scheduling have attracted much attention due to their ability to provide solutions from a global point of view. However, current approaches which focus only on a single objective with the aim of minimizing the total delay and the number of conflicts, cannot provide controllers with variety of optional solu-tions, representing different trade-offs. Furthermore, the flight track error is often overlooked in the current research. Therefore, in order to make the model more realistic, in this paper, we formulate the long-term conflict avoidance problem as a multi-objective optimization problem, which minimizes the total delay and reduces the number of conflicts simultaneously. As a complex air route network needs to accommodate thousands of flights, the problem is a large-scale combinatorial optimization problem with tightly coupled variables, which make the problem difficult to deal with. Hence, in order to further improve the search capability of the solution algorithm, a cooperative co-evolution (CC) algorithm is also introduced to divide the complex problem into several low dimensional sub-problems which are easier to solve. Moreover, a dynamic grouping strategy based on the conflict detection is proposed to improve the optimization efficiency and to avoid premature convergence. The well-known multi-objective evolution-ary algorithm based on decomposition (MOEA/D) is then employed to tackle each sub-problem. Computational results using real traffic data from the Chinese air route network demonstrate that the proposed approach obtained better non-dominated solutions in a more effective manner than the existing approaches, including the multi-objective genetic algorithm (MOGA), NSGAII, and MOEA/D. The results also show that our approach provided satisfactory solutions for controllers from a practical point of view.
\end{abstract}

Keywords Air traffic management; Conflict avoidance; Combinatorial optimization; Multi-objective; Cooperative co-evolution

\footnotetext{
Citation Guan X M, Lv R L, Zhang X J, Chen J, Weiszer M. . Title for citation. Sci China Inf Sci, for review
}

\section{Introduction}

In the recent years, the sharp increase in air traffic flow has reached the limits of airspace capacity which caused the air traffic congestion to become a more serious issue [1,2]. As a result, the key airports and trunk routes of many countries and areas are facing a highly complicated traffic situation. In the

\footnotetext{
* Corresponding author (email: zhxj@buaa.edu.cn)
} 
local high-density operation, the safe sep-aration among aircraft is often difficult to keep, which leads to conflict situations and near-misses frequently. Fur-thermore, the air route network is very complicated with thousands of waypoints, air route segments and flights op-erations. On each air route segment, there are many flight levels in altitude height with about 300 meters separation required for flights of different directions to avoid head-to-head conflict. With the rapid increase of air travel demand, the current airspace is becoming increasingly crowded and thus the conflict probability between aircraft especially at cross waypoints could dramatically rise. Along with the above mentioned problems, as the air traffic sys-tem is a tightly coupled and large-scale system with traffic flows intersecting each other, conflicts tend to spread within it, which not only impairs the safety, but also restricts sus-tained development of air transportation [3].

Conflict resolution approaches play a very important role in keeping a safe airspace. However, as the current sector-based air traffic system still cannot provide accurate traffic surveillance information covering a huge airspace, it is difficult to fully predict long-term conflicts and thus make decisions in advance to avoid them. As a result, cur-rent approaches are mainly focused on short-term conflict avoidance, which can efficiently solve conflicts in a rela-tively small short time window [4]. During the last decades, many approaches have been proposed, which can be mainly categorized into: rule-based methods [5], game the-ory methods [6,7], field methods [8], geometric methods [9], numerical optimization methods [10-12], and multi-agent methods [13-15].

However, as the increase in air traffic flow continues, the above conflict resolution approaches cannot provide good solutions in terms of both effectiveness and timeliness due to the new features of the optimization problem, such as large scale, high complexity and tightly coupled variables. Moreover, without full consideration of the overall situation, providing short-term ad hoc solutions for flights could lead to a knock on effect due to the tight coupling between flights, which would jeopardize airspace safety [3].

In the recent years, the FAA and Eurocontrol proposed the concept of $4 \mathrm{D}$-Trajectory (4DT) as the operation foundation of future air traffic management, which defines a flight trajectory using three spatial dimensions plus one time dimension. As the development of the advanced technology continues, flights can be accurately described in both space and time, which can significantly reduce the uncertainty of the flight trajectory. According to the initial operational experiment of the Eurocontrol, the uncertainty to all the waypoints of a flight path can be controlled within about 10 seconds. Most uncertainty will be eliminated through the adjustment of a flight, such as instant velocity change. As a result, the air traffic control can be realized with the current traffic situation and its evolutionary trend in a huge airspace. This also provides an operational and technical support for long-term management. Subsequently, the long-term conflict avoidance (LCA) method supporting 4DT operation has drawn much attention of researchers and practitioners from air traffic management domain, and it is envisioned as a key technology which can address the challenges caused by increased air traffic flow in the future $[16,17]$.

Considering thousands of flights in a complex air route network, the LCA problem is a large-scale combinatorial optimization problem with tightly coupled decision varia-bles, as well as complicated constraints which make it dif-ficult to solve by classical approaches. Therefore, an evolu-tionary algorithm (EA) is adopted [16]. A sliding forecast time window is introduced to reduce the dimension of the problem in order to obtain feasible solutions. However, it may overstock the large amount of flights in later time windows, causing a high difficulty for the EA-based ap-proach to solve. Recently, a cooperative co-evolution (CC) strategy has been successfully used to handle the problem [18]. It uses a divide-andconquer strategy to decompose the large-scale problem into several sub-problems which are easier to be solved. In the CC-framework, the grouping strategy is a critical step especially for this large-scale com-plex problem. In order to improve the optimization effi-ciency, some other problem decomposition methods have been proposed, such as the splitting-in-half grouping [19], the correlation-based adaptive variable partitioning [20], the delta grouping [21], and the dependency identification technique [22]. Although these decomposition methods are effective in generic optimisation problems, they cannot take full advantage of the prior knowledge in order to minimise the interdependencies of the variables for the LCA problem. 
Recently, with the aim to minimize the risk of premature convergence, a memetic algorithm (MA) is adopted [3]. It utilizes a specially designed local search operator and an adaptive local search frequency strategy to improve search capability of the algorithm. However, these previous works neglected the track error of flights, which makes them im-practical. Furthermore, they considered the minimization of the aggregated flight delay and conflicts as a single objec-tive [17]. While, in the real operation, controllers often try to seek a good trade-off between the flight delay and the number of conflicts.

In light of the above issues, in this paper, the conflict sit-uation in the waypoint network is evaluated with consider-ation of track error of flights to make the model more prac-tical and realistic. In order to incorporate more objectives, we formulate the long-term conflict avoidance problem as a multi-objective optimization problem, which can mini-mize the total delay and reduce the number of conflicts simultaneously. To further improve the search capability of the algorithm, a cooperative co-evolution algorithm is in-troduced to divide the complex problem into several low dimensional sub-problems [23]. Furthermore, a dynamic grouping strategy based on the conflict between flights is designed to improve search efficiency and to avoid prem-ature convergence. The well-known multi-objective evolu-tionary algorithm based on decomposition (MOEA/D) is then employed to tackle each sub-problem separately [24]. Computational results using real traffic data from the Chi-nese air route network demonstrate that the proposed ap-proach achieved better non-dominated solutions in a more efficiently manner than the existing approaches, such as the multi-objective genetic algorithm (MOGA) [25], NSGAII [26], or MOEA/D. The results also show that our approach can provide satisfactory solutions for controllers in a more practical sense.

The rest of this paper is organized as follows. Firstly, the problem is formulated in Section 2. Section 3 presents the details of our solution approach. The results of computa-tional experiments are presented and analyzed in Section 4. Finally, some conclusions and future research directions are drawn in Section 5 .

\section{Problem formulation}

The problem described in this paper can be formulated as follows. Let $\mathrm{W}$ denote the set of waypoints in the consid-ered airspace, then the waypoint sequence of the trajectory of flight i is $\left\{W_{j}{ }^{i}\right\}_{j=0, \ldots, n w_{i}} W_{j}{ }^{i} \in \mathbb{R}^{2}$ where $\mathrm{j}$ is the index of the waypoint in the sequence, nwi is the number of waypoints in the path of flight i. There are $\mathrm{n}$ flights $\left(F_{1}, F_{2} \ldots F_{n}\right)$ in total with specific flight plans. The velocity of flight $\mathrm{i}$ in each segment is $\left\{V_{j}{ }^{i}\right\}_{j=0, \ldots, n w_{i}}, V_{j}^{i} \in \mathbb{R}_{+}{ }^{2}$. Without consideration of the track error, the estimated arrival time at each waypoint of fight i can be obtained by [27]:

$$
T_{j}^{i}=\frac{\left\|W_{j}^{i}-W_{j-1}^{i}\right\|}{v_{j}^{i}}+T_{j-1}^{i}, j=1, \ldots, n w_{i}
$$

where $T_{0}{ }^{i}=0$ and $W_{0}{ }^{i}$ is the first waypoint of the path of flight i. The flight dis-tance s of flight $\mathrm{i}$ at time $\mathrm{t}$ is:

$$
s^{i}(t)=v_{j}^{i}\left(t-T_{j-1}^{i}\right)+s^{i}\left(T_{j-1}^{i}\right), t \in\left(T_{j-1}^{i}, T_{j}^{i}\right]
$$

The current position $\mathrm{p}$ of flight $\mathrm{i}$ at time $\mathrm{t}$ is:

$$
p^{i}(t)=p^{i}\left(T_{j-1}^{i}\right)+v_{j}^{i}\left(t-T_{j-1}^{i}\right) \frac{\left(W_{j}^{i}-p^{i}\left(T_{j-1}{ }^{i}\right)\right)}{\left\|W_{j}^{i}-p\left(T_{j-1}{ }^{i}\right)\right\|}
$$

where $s^{i}\left(T_{0}{ }^{i}\right)=0$, and $p^{i}\left(T_{0}{ }^{i}\right)=W_{0}{ }^{i}$.

Under the operation of the sector-based air traffic man-agement, the track error of flights in general obeys a Gaussian distribution where the mean is zero, and the hori-zontal standard deviation $\delta_{s}^{2}$ is defined by:

$$
\delta_{s}^{2}(t) \sim r_{s}^{2} t^{2}
$$


Xiangmin Guan, et al., et al. Sci China Inf Sci 4

and the lateral standard deviation is described by:

$$
\delta_{c}^{2}(t) \sim \min \left\{r_{c}^{2} s^{2}(t), \bar{\delta}_{c}^{2}\right\}
$$

where $\bar{\delta}_{c}^{2}$ is the maximum of the lateral standard deviation. We can see that the horizontal standard deviation and the lateral standard deviation will increase as the time grows, and generally $\delta_{s}(t)$ is larger than $\delta_{c}(t)$. In addition, the vertical standard deviation is a constant.

However, under the operation of $4 \mathrm{D}$ trajectory, the ac-curacy of the flight path could be greatly improved. More-over, with the help of the flight management system, flights can arrive at each waypoint with higher precision. There-fore, in this paper, both the horizontal standard deviation and the lateral standard deviation are considered to be constant and are defined by $\delta_{s}$ and $\delta_{c}$ respectively. In addition, the estimated arrival time at each waypoint is assumed to obey a Gaussian distribution with zero mean and $\delta_{t w}$ as the standard deviation.

Suppose that the angle between the current velocity of flight $\mathrm{i}$ and $\mathrm{x}$ axis is $\theta_{j}$ in the plane coordinate system, and in the body coordinate system it can be denoted by

$$
R\left(\theta_{j}\right)=\left(\begin{array}{cc}
\cos \theta_{j} & -\sin \theta_{j} \\
\sin \theta_{j} & \cos \theta_{j}
\end{array}\right)
$$

Hence, the predicted position of flight $\mathrm{i}$ at time $\mathrm{t}$ can be obtained by

$$
X^{i}(t)=p^{i}\left(T_{j-1}{ }^{i}\right)+v_{j}^{i}\left(t-T_{j-1}^{i}+\delta_{t w}^{2}\right) \frac{\left(W_{j}^{i}-p^{i}\left(T_{j-1}{ }^{i}\right)\right)}{\left\|W_{j}^{i}-p\left(T_{j-1}{ }^{i}\right)\right\|}+D
$$

where $\mathrm{D}$ is a covariance matrix, and $D=R(\theta) \bar{D} R(\theta)^{T}$, with $\bar{D}=\left(\begin{array}{cc}\delta_{s}^{2} & \\ & \delta_{c}^{2}\end{array}\right)$ if

$$
C D=v_{j} \frac{\left(W_{j}^{i}-p^{i}\left(T_{j-1}{ }^{i}\right)\right)}{\left\|W_{j}^{i}-p\left(T_{j-1}{ }^{i}\right)\right\|} \delta_{t w}^{2}+D
$$

Then, $X^{i}(t)$ can be defined by

$$
X^{i}(t) \sim N\left(P^{i}(t), C D\right)
$$

Considering the flight set $\mathrm{F}$ in a time window, the dis-tance function between any two flights $\mathrm{i}$ and $\mathrm{j}$ is denoted by

$$
\operatorname{dist}_{i j}(t)=\left\|X_{i}(t)-X_{j}(t)\right\|
$$

It is assumed that the positions of flights are not relevant, so dist ${ }_{i j}(t)$ obeys a Gaussian distribution as follows:

$$
\operatorname{dist}_{i j}(t) \sim N\left(P^{i}(t)-P^{j}(t), 2 C D\right)
$$

Then, the conflict probability $P C_{i j}(t)$ of two flights $\mathrm{i}$ and $\mathrm{j}$ at time $\mathrm{t}$ can be computed by

$$
P C_{i j}(t)=\int_{\text {dist }_{i j}<\varepsilon_{i j}} p_{i j}^{d_{t}}(y) d y
$$

where $p_{i j}^{d_{t}}(y)$ is the probability density function of $d_{i s t}(t)$. The Conflict Situation (CS) of all flights in the considered airspace can be defined by

$$
C S=\sum_{i=1}^{n} \sum_{j>i}^{n} M P C_{i j}
$$

where MPCij is the maximum conflict probability of two flights, and it can be described by

$$
M P C_{i j}=\underset{t \in\left[T_{i j}^{1}, T_{i j}^{2}\right]}{M A X}\left(P C_{i j}(t)\right)
$$


Hence, the first objective is formulated to minimize the total maximum conflict probability, and it can be defined by

$$
\operatorname{Min} f_{1}=C S
$$

In this work, the ground delay method is used to avoid conflict at waypoints, which is an effective way by delaying flights while they are still on the ground before departure. However, in order to reduce the cost for airlines, the sum of flight delays is formulated as the second objective which is defined by

$$
\operatorname{Min} f_{2}=\frac{1}{n} \sum_{i}^{n} \delta_{i}
$$

where $\delta_{i}$ presents the departure delay of flight $\mathrm{i}$, and $\delta_{i} \in\left[\begin{array}{ll}0 & \delta_{\max } / t s\end{array}\right]$, where $\delta_{\max }$ is the maximum allowable delay. It means that the delay of any flight is limited by a maximum value in order to prevent some flights being postponed for too long. ts is the time step for time sampling.

It can be demonstrated that the LCA problem is a large-scale combination optimization problem with two objectives. Furthermore, the variables and constraints are tightly coupled because of conflict avoidance.

\section{Optimization Framework}

In order to solve the abovementioned optimization problem in an efficient manner and to avoid premature convergence, an efficient multi-objective optimization framework is proposed in this section. Firstly, a cooperative co-evolution (CC) algorithm is introduced to divide the complex problem into several low dimensional sub-problems. Towards this aim, a dynamic grouping strat-egy based on the conflict between flights is designed as a heuristic strategy. Then, the well-known multi-objective evolutionary algorithm based on decomposition (MOEA/D) is employed to solve each sub-problem. The framework is described in Figure 1.

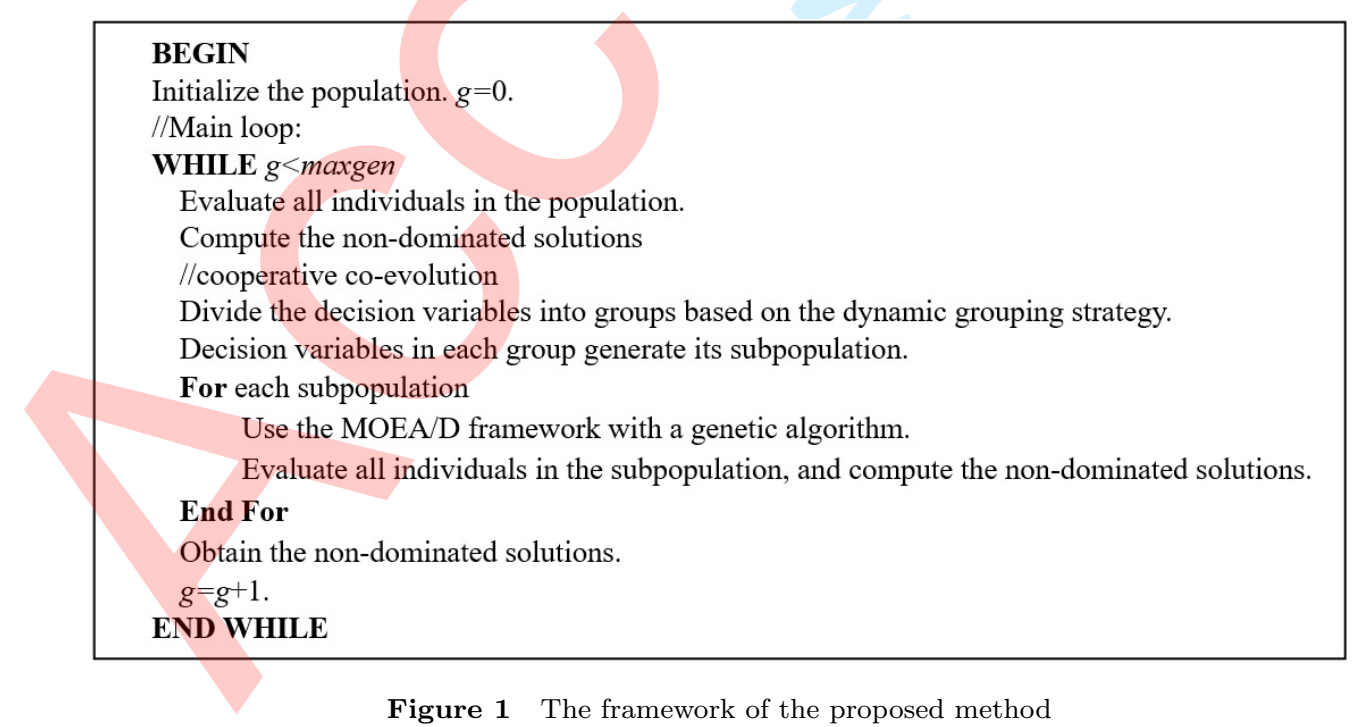

In the following subsections, some important mecha-nisms, such as the dynamic grouping strategy, subcompo-nent optimization, adaptive crossover, and mutation oper-ators are elaborated in more details.

\subsection{The Dynamic Grouping Strategy}

The cooperative co-evolution algorithm has two critical steps. In this section, we mainly describe the dynamic grouping strategy which is used to divide flights into groups based on conflicts. 
Xiangmin Guan, et al., et al. Sci China Inf Sci 6

In order to describe if two flights conflict with each other, a matrix C [28] is adopted in this work as defined below.

$$
C=\left(\begin{array}{ccc}
C_{11} & \cdots & C_{1 n} \\
\vdots & \vdots & \vdots \\
C_{n 1} & \cdots & C_{n n}
\end{array}\right)
$$

where

$$
C_{i j}=\left\{\begin{array}{l}
1, \text { if } F_{i} \text { and } F_{j} \text { conflict }, i \neq j \\
0 . \text { otherwise }
\end{array}, i, j=1, \ldots, n\right.
$$

Firstly, if there is no conflict among any two flights, the random grouping strategy will be employed, which ran-domly divides the flights into ns groups with the same size.

Secondly, if there are at least two flights which conflict with each other, i.e.,

$$
\exists i \neq j, \text { st } \quad C_{i j}=1
$$

then, the flights are divided into sn groups based on the dynamic grouping strategy which can be defined by

$$
\text { group }_{k}=\left(F_{k}^{(1)}, F_{k}^{(2)}, \ldots, F_{k}^{\left(m_{k}\right)}\right), 1 \leqslant k \leqslant s n, 1 \leqslant m_{k}<n, \sum_{k=1}^{s n} m_{k}=n .
$$

where $F_{k}^{(j)}$ denotes the jth flight in group $_{k}$ and $m_{k}$ indicates the number of flights in group $_{k}$.

The flights in each group satisfy

$$
\forall a \in \operatorname{group}_{k}, \forall b \in \text { group }_{l}, \text { st }, C_{a b}=0
$$

and flights from different groups satisfy

$$
\forall a \in \text { group }_{k}, \forall b \in \text { group }_{l}, \text { st }, C_{a b}=0
$$

\subsection{Subcomponent Optimization}

In this work, the fast GA is proposed as the global search method [28].

Another critical point is the optimization of each group. In this paper, a fast GA is incorporated into the MOEA/D framework.

The sub-population of each group includes ps individuals indicating the possible solutions of flights in this group. Hence the sub-population is a matrix defined by

$$
\text { subpop }_{k}=\left\{f_{k}^{(1)}, f_{k}^{(2)}, \ldots, f_{k}^{(p s)}\right\}, 1 \leqslant k \leqslant s n,
$$

where $f_{k}^{(i)}(1 \leqslant i \leqslant p s)$ s a vector which can be defined by

$$
f_{k}^{(i)}=\left(\delta_{k}{ }^{(i 1)}, \delta_{k}{ }^{(i 2)}, \ldots, \delta_{k}{ }^{\left(i m_{k}\right)}\right), 1 \leqslant m_{k}<n, \sum_{k=1}^{s n} m_{k}=n
$$

where $\delta_{k}{ }^{(i j)}$ denotes the delay time slot of flight $F_{k}{ }^{(j)}$ of chromosome $\mathrm{j}$ in group $_{k}$.

The general framework of MOEA/D [24] is shown in Fig-ure 2.

\subsubsection{Adaptive crossover and mutation operators}

The adaptive crossover and mutation operators are spe-cially designed for the LCA problem based on the fitness of each gene in the individual. The fitness takes the ground delay and conflict probability of flights into account. The fitness of each flight in group $_{k}$ is defined by

$$
f i t_{k}^{j}=\frac{1-\delta_{k}^{j} / \delta_{\max }}{1+c s_{k j}},\left(1 \leqslant j \leqslant m_{k}\right)
$$


where $c s_{k j}$ is the total conflict probability of flight $\mathrm{j}$ with other flights.

The mechanism of the adaptive crossover is shown in Fig-ure 3. In this example, A and B are parents in sub-population k. If $f i t_{k}^{A_{1}}>f i t_{k}^{B_{1}}$, the two children will inherit from $A_{1}$ accordingly, and if $f i t_{k}^{B_{1}}>f i t_{k}^{A_{1}}$ , they in-herit from $B_{1}$. Otherwise, the genes of children are obtained in a way as follows

$$
\begin{aligned}
& C A_{1}=\text { floor }\left(\alpha A_{1}+(1-\alpha) B_{1}\right), \\
& C B_{1}=\text { floor }\left(\alpha B_{1}+(1-\alpha) A_{1}\right) .
\end{aligned}
$$

where $\alpha$ is the parameter of the linear combination.

For adaptive mutation operator, as can be seen from Figure 4 , if $g f_{k}^{j}<\varepsilon$, the gene $\mathrm{j}$ mutates with a probability of $p_{k}$.

\section{Experimental studies}

\subsection{Database and Experimental Setup}

The national route network of China consists of 1706 air route segments, 940 waypoints and 150 airports as shown in Figure 2. The air traffic data was obtained from Civil Aviation Administration of China (CAAC) for a whole day of 7 October, 2009. It is worth mentioning that the takeoff and landing phases of flights are truncated within a given radius (usually $10 \mathrm{NM}$ ) around airports. The traffic around airports is managed following specific procedures imposed by the Terminal Control Area (TCA) control services in these zones.
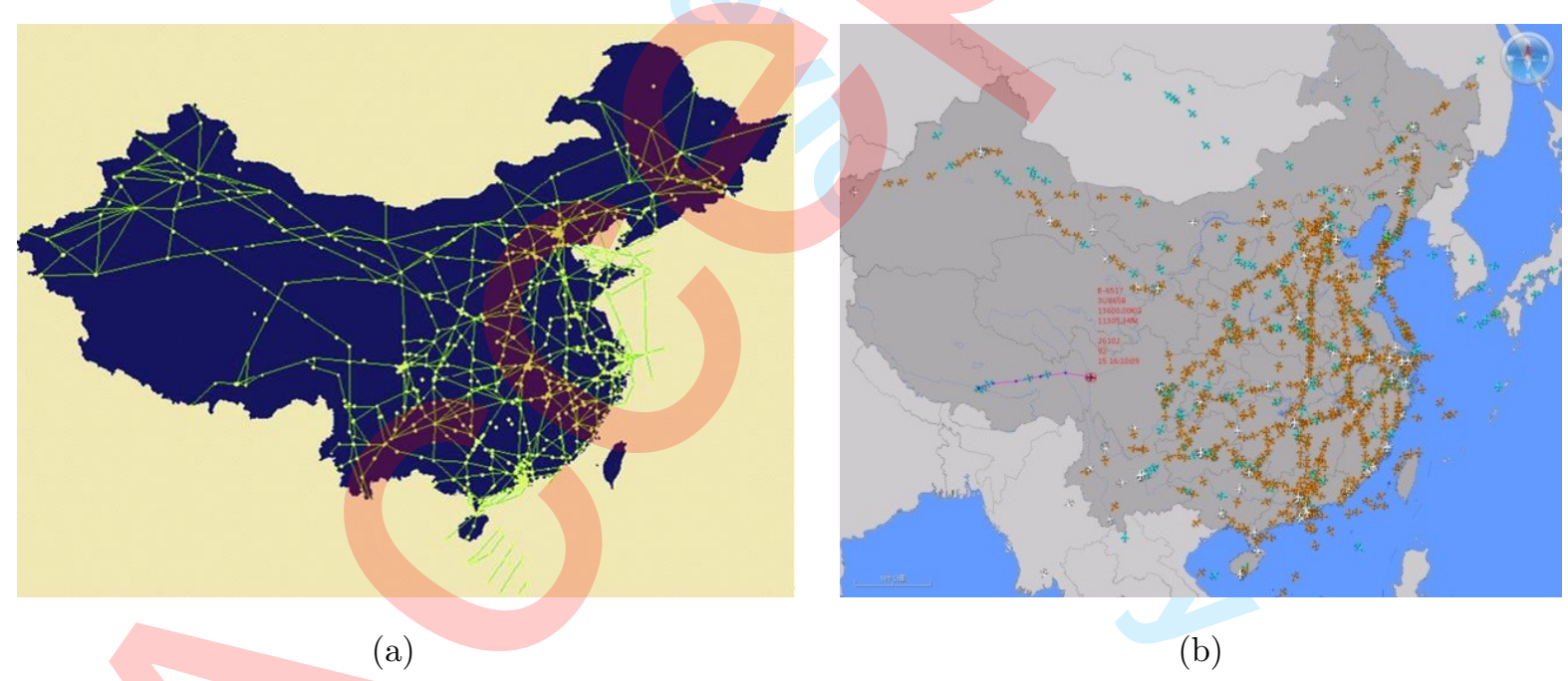

(b)

Figure 2 (a) The airway point network in China. (b) Flights operation in China.

The minimum safe time interval is equal to $=60 \mathrm{~s}$. $\delta_{\max }$ is set to be $90 \mathrm{~min}$, the value interval of $\delta$ is $0.25 \mathrm{~min}$, and $\varepsilon=0.3$.

In order to compare with the proposed MOCC, MOEA/D, MOGA (Delahaye et al. 2005), NSGA2 (Deb et al. 2002) are selected, and all these algorithms were implemented in $\mathrm{C}++$ in this work. Computational experiments were carried out on a computer with an E5620 2.4GHz CPU with 12GB RAM. For each algorithm, the results were collected and analyzed based on 15 independent runs.

The parameters used in all experiments are listed in Table 1, and they are often adopted in other algorithms [18].

\subsection{The depiction of conflict situation}

Next, the relationship between the number of flights and the conflict situation in the considered airspace is depicted in Figure 6. We can see that there are about 1000 flights during every two hours in Figure 6(a). 


\section{Input:}

(1) A stopping criterion.

(2) $n p$ : the number of the sub-problems.

(3) An uniform spread of $n$ weight vectors: $\lambda^{1}, \ldots, \lambda^{n p}$.

(4) $T$ : the number of the weight vectors in the neighborhood of each weight vector. Output: Approximation to the PF and PS.

\section{Procedure:}

Step 1 Initialization:

Step 1.1 Compute the Euclidean distances between the weight vectors and work out the $T$ closest weight vectors to each weight vector. For each $i=1, \cdots, n p$, set $B(i)=\left\{i_{1}, \cdots, i_{T}\right\}$, where $\lambda^{i_{1}}, \cdots, \lambda^{i_{T}}$ are the $T$ closest weight vectors to $\lambda^{i}$.

Step 1.2 Generate an initial population $x^{1}, \cdots, x^{p p}$. Calculate the fitness values of the population.

Step 1.3 Initialize $z=\left(z_{1}, \cdots, z_{m}\right)$, where $z_{j}=\min _{1 \leq i \leq n} f_{j}\left(x^{i}\right)$.

\section{Step 2 Update:}

For $i=1, \cdots, n p$,

Step 2.1 Selection of the mating pool:

Generate a random number which is uniformly distributed in $[0,1]$. Set

Step 2.2 Reproduction:

$$
P=\left\{\begin{array}{l}
B(i), \quad \text { if rand }<\delta \\
\{1, \ldots, \text {, } p \text { otherwise }
\end{array}\right.
$$

Set $r_{l}=i$, and randomly select two indexes $k, l$ from $P$, and then generate a new solution $y$ using mutation and crossover operators of GA.

Step 2.3 Repair:

If an element of $y$ is out of the bound of $\Omega$, its value is reset to be a randomly selected value inside the boundary.

Step 2.4 Update of the reference point: For each $j=1, \cdots, m$, if $z_{j}>f_{j}(y)$, then set $z_{j}=f_{j}(y)$.

\section{Step 2.5 Replacement of solutions}

Step 3 Stopping Criterion:

If the stopping criteria is satisfied, then stop the algorithm and output PF and PS. Otherwise, go to Step 2.

Figure 3 Algorithmic flow of MOEA/D with GA.

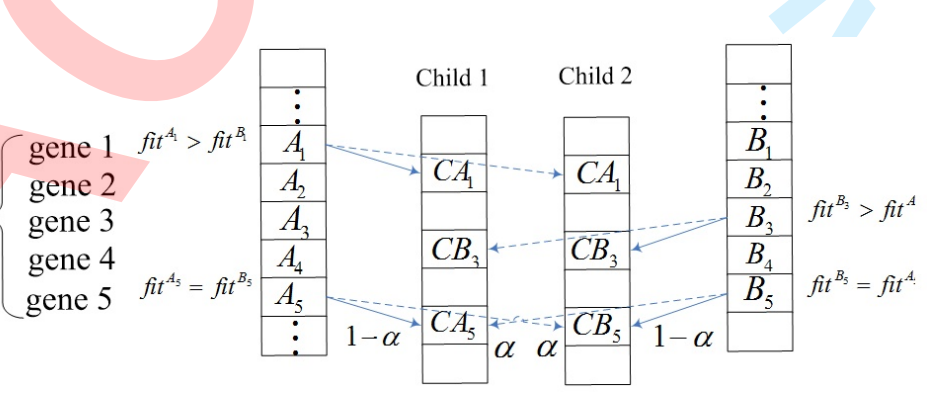

Figure 4 Adaptive crossover operator.

Table 1 Parameters of the experiments.

\begin{tabular}{ccc}
\hline Parameters & Description & Value \\
\hline $\mathrm{ps}$ & Population size & 100 \\
maxgen & Max generation & 500 \\
$\mathrm{p}_{c}$ & Crossover probability & 0.8 \\
$\mathrm{p}_{m}$ & Mutate probability & 0.1 \\
\hline
\end{tabular}




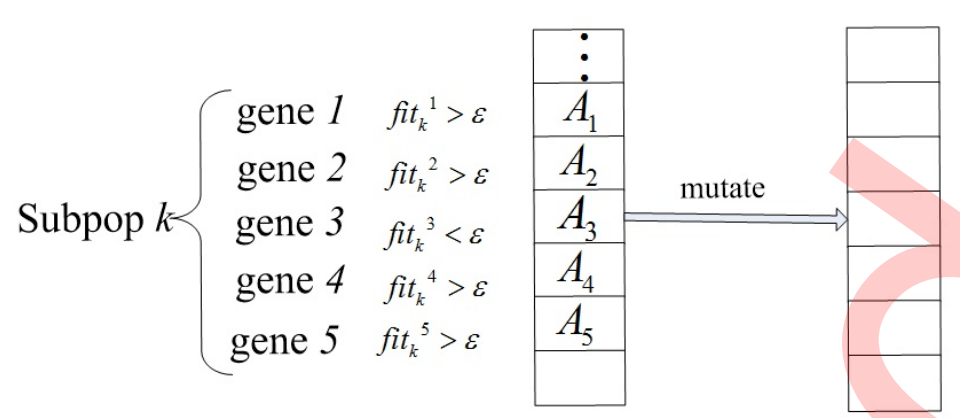

Figure 5 Adaptive mutate operator.

The number of flights from $7 \mathrm{AM}$ to $9 \mathrm{AM}$ is the largest. The total maximum conflict probability of all flights in each time period is about 300. In addition, in Figure 6(b), it can be seen that as the number of flights grows, the total maximum conflict probability increases quickly.

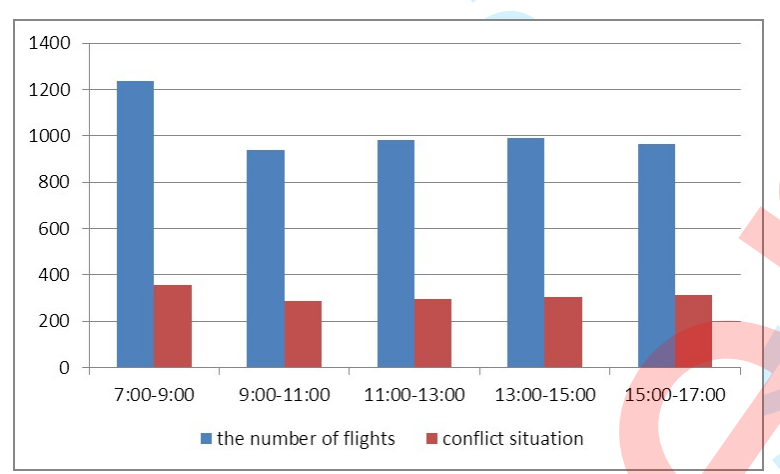

(a)

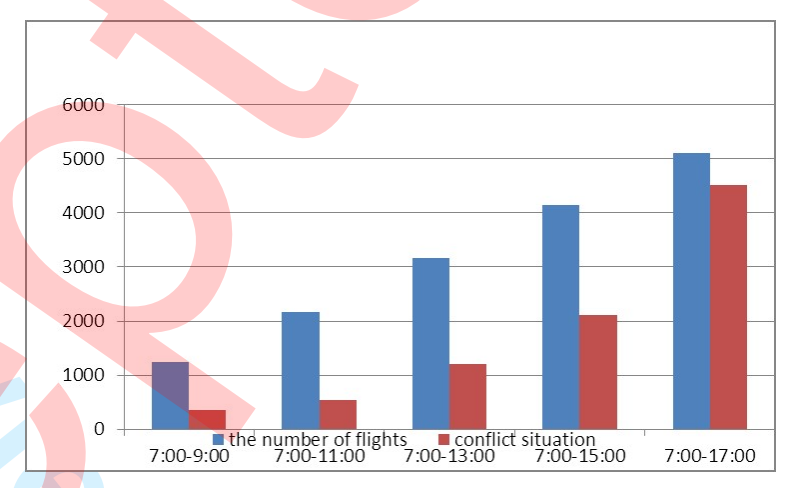

(b)

Figure 6 The relationship between the number of flights and the conflict situation in the considered airspace.

\subsection{Comparison with the existing methods}

In order to compare the performance of the abovemen-tioned algorithms, two scenarios including 960 flights (rep-resent the busiest one hour) and 1664 flights (represent the busiest three hours) are considered. In addition, three typi-cal metrics are adopted to evaluate the performance of the solutions obtained by each of the algorithms. The conver-gence metric $(\gamma)[26]$, the spread metric $(\Delta)$ [29], and the Hypervolume metric $I_{H}$ is used $[30,31]$.

Table 2 and Table 3 summarize the average values of $I_{H}, I_{D}$ and $\Delta$ over 15 independent runs. The best results are highlighted in boldface in each row of the table. We can see from both tables that the proposed algorithm outperforms the other three algorithms in all metrics. Moreover, when the number of flights increases, it performs even better. There-fore, it is concluded that MOCC has superiority in solving large-scale problems such as the one in this paper.

Table 2 Comparison of different algorithms for 960 flights $\left(I_{H}, \gamma, \Delta\right)$.

\begin{tabular}{cccc}
\hline Algorithms & $I_{H}$ & $\gamma$ & $\Delta$ \\
\hline MOGA & 3994 & 98.99 & 1.313 \\
NSGA2 & 5468 & 68.93 & 1.213 \\
MOEA/D & 6378 & 55.23 & 1.263 \\
MOCC & 6731 & 43.63 & 1.010 \\
\hline
\end{tabular}

Additionally, the non-dominated solutions with the least delay time cost (DTC) and with the least conflict situation (CS) obtained by the all algorithms over 15 runs are listed in Tables 4 and 5 under 
Xiangmin Guan, et al., et al. Sci China Inf Sci 10

Table 3 Comparison of different algorithms for 1664 flights $\left(I_{H}, \gamma, \Delta\right)$.

\begin{tabular}{cccc}
\hline Algorithms & $I_{H}$ & $\gamma$ & $\Delta$ \\
\hline MOGA & 4119 & 86.30 & 1.1751 \\
NSGA2 & 5249 & 56.73 & 1.2426 \\
MOEA/D & 5951 & 63.30 & 1.3448 \\
MOCC & 6467 & 57.45 & 0.8181 \\
\hline
\end{tabular}

the two scenarios separately. It can be observed that both of the non-dominated solutions with the least DTC and the least CS obtained by MOCC are not dominated by the corresponding solutions of the other three algorithms when the number of flights is 960 . In indeed, in most comparisons under this scenario, MOCC provides better solutions in both objectives. The same conclusion applies to the scenario when the number of flights is 1664 .

Figure 7 shows the non-dominated solutions obtained by respective algorithms. Specifically, the nondominated solu-tions of each algorithm were obtained over 15 runs. FromFigure 7, it can be concluded that MOCC performs the best because its solutions dominate those obtained by other algorithms. Among all algorithms, MOGA has the worst performance in terms of convergence. MOEA/D performs better than NSGA2 in terms of convergence and diversity.

From the experimental results, we conclude that MOCC performs better than the other three methods for both sce-narios. MOCC adopts an effective multi-objective optimi-zation framework based on the CC (i.e. dynamic grouping) and MOEA/D, greatly improving its the search capability. The CC divides the complex problem into several low-dimension sub-problems, which makes the problem easier to solve. The sub-problems work cooperatively to obtain better solutions. Furthermore, the CC takes full ad-vantage of the characteristics of the long-term conflict avoidance problem and is based on the conflict among flights, leading to improved search efficiency. The im-proved search performance is also due to the employment of the well-known multi-objective evolutionary algorithm based on decomposition (MOEA/D) to solve each sub-problem.

\subsection{Comparison between dynamic grouping strategy and other popular strategies}

The experiment in this section is designed to further in-vestigate the contribution of the proposed dynamic group-ing strategy. The grouping strategy is a key issue in the CC-based framework. There are several popular grouping strategies, e.g. one-dimensional grouping strategy, split-ting-in-half grouping strategy, and random grouping strate-gy [19]. In the following, two of these grouping strategies are compared with the proposed one. All grouping strategies are implemented within the same CC based framework and share exactly the same settings. The two grouping strategies for comparison are briefly described as follows:

- Splitting-in-half based strategy (SIH): Each sub-group contains half of the total aircraft.

- Random grouping strategy (RG): All the aircraft are randomly divided into several sub-groups.

Table 4 Non-dominated solutions with the least CS and the least DTC for 960 flights.

\begin{tabular}{ccccc}
\hline Algorithms & \multicolumn{2}{c}{ Solutions with least CS } & \multicolumn{2}{c}{ Solutions with least DTC } \\
& CS & DTC & CS & DTC \\
\hline MOGA & 62.02 & 18.76 & 140.6 & 9.552 \\
NSGA2 & 22.52 & 20.00 & 138.9 & 6.183 \\
MOEA/D & 15.17 & 13.40 & 109.4 & 3.256 \\
MOCC & 0.3841 & 15.42 & 108.5 & 2.692 \\
\hline
\end{tabular}

Table 6 and Table 7 show the average value of $I_{H}, I_{D}$ and $\Delta$ over 15 independent runs of the algorithms for respective scenarios. The best value is highlighted in boldface in each row of the table. It can be concluded from both tables that the proposed algorithm outperforms the other three algo-rithms in terms of $I_{H}, I_{D}$ and $\Delta$. Hence, the dynamic group-ing strategy has superiority in solving large-scale problems such as the one in this paper. 
Table 5 Non-dominated solutions with the least CS and the least DTC for 1664 flights.

\begin{tabular}{ccccc}
\hline Algorithms & \multicolumn{2}{c}{ Solutions with least CS } & \multicolumn{2}{c}{ Solutions with least DTC } \\
& CS & DTC & CS & DTC \\
\hline MOGA & 52.67 & 21.12 & 178.2 & 8.873 \\
NSGA2 & 17.91 & 22.46 & 101.3 & 6.854 \\
MOEA/D & 31.21 & 14.03 & 137.3 & 3.202 \\
MOCC & 0.4173 & 15.44 & 116.2 & 2.734 \\
\hline
\end{tabular}

Table 6 Comparison of different algorithms for 960 flights $\left(I_{H}, \gamma, \Delta\right)$.

\begin{tabular}{cccc}
\hline Algorithms & $I_{H}$ & $\gamma$ & $\Delta$ \\
\hline MOCC-SIH & 5534 & 61.23 & 1.161 \\
MOCC-RG & 6028 & 54.38 & 1.072 \\
MOCC & 6731 & 43.63 & 1.010 \\
\hline
\end{tabular}

Table 7 Comparison of different algorithms for 1664 flights $\left(I_{H}, \gamma, \Delta\right)$.

\begin{tabular}{cccc}
\hline Algorithms & $I_{H}$ & $\gamma$ & $\Delta$ \\
\hline MOCC-SIH & 5370 & 62.68 & 1.1754 \\
MOCC-RG & 5875 & 60.45 & 1.0864 \\
MOCC & 6467 & 57.45 & 0.8181 \\
\hline
\end{tabular}


The non-dominated solutions with the least delay time cost (DTC) and the least conflict situation (CS) obtained by all algorithms over 15 runs are listed in Tables 9 and 10. We can see that under both scenarios, the dynamic grouping strategy performs the best in both objectives.

The splitting-in-half grouping strategy cannot cope with this large-scale problem with more than half flights in each group still vulnerable to potential conflicts. Although the random grouping strategy can reduce such potential con-flicts in the case of the two interacting flights in the same group, its performance will drop dramatically when there are more than two interacting flights In general, the split-ting-in-half and random grouping strategies represent a blind search mechanism and are more easily to be trapped in a local optimum. On the contrary, the proposed dynamic grouping strategy exploits the pattern reflected in potential conflicts among flights leading to an improved global search capability.

\subsection{Application to real operations}

In this section, we further investigate the applicability of the proposed approach in real operations, i.e. its ability to provide feasible solutions for the air traffic controllers to keep safe separation of flights.

It is worth mentioning that the proposed method in this paper is a pre-tactical approach which can be used to solve conflicts that happen in a time scale from several hours to a few days in advance. Therefore, we do not consider dis-turbances. More specifically, the computational time needed to get feasible solutions of MOCC is about 5 and 17 minutes for scenario with 960 and 1664 flights respectively. This is sufficient for a real pre-tactical application. About 30 non-dominated solutions in scenario 1 and 20 non-dominated solutions in scenario 2 are obtained. Prac-tically, controllers may only need a few feasible solutions. Therefore, the computation time can be much shorter The computation time can be further reduced using more ad-vanced parallel computation technology.

We also noticed that even for the scenario with 1664 flights, the average number of conflicts using MOCC is al-most 0 and the average delay can be controlled within 15 minutes. Furthermore, as can be seen from Figure 7, when the average delay is within 10 minutes, the maximum number of flights will be under 20 which can be comforta-bly handled by air traffic controllers.

In conclusion, the proposed MOCC can largely improve the optimization capability and avoid local optima. It rep-resents the best search and grouping strategy among all solution approaches dealing with the long-term conflict avoidance problem. Although the current version of MOCC cannot be applied to a real time application, it is sufficient for a pre-tactical management application.

Table 8 Non-dominated solutions with the least CS and the least DTC for 960 flights.

\begin{tabular}{|c|c|c|c|c|}
\hline \multirow[t]{2}{*}{ Algorithms } & \multicolumn{2}{|c|}{ Solutions with least CS } & \multicolumn{2}{|c|}{ Solutions with least DTC } \\
\hline & $\mathrm{CS}$ & DTC & $\mathrm{CS}$ & DTC \\
\hline MOCC-SIH & 58.67 & 17.47 & 154.4 & 8.754 \\
\hline MOCC-RG & 10.54 & 16.98 & 113.5 & 4.785 \\
\hline MOCC & 0.3841 & 15.42 & 108.5 & 2.692 \\
\hline \multirow[t]{2}{*}{ Algorithms } & \multicolumn{2}{|c|}{ Solutions with least CS } & \multicolumn{2}{|c|}{ Solutions with least DTC } \\
\hline & $\mathrm{CS}$ & DTC & $\mathrm{CS}$ & DTC \\
\hline MOCC-SIH & 64.35 & 24.98 & 189.2 & 9.358 \\
\hline MOCC-RG & 12.57 & 23.47 & 164.2 & 7.426 \\
\hline MOCC & 0.4173 & 15.44 & 116.2 & 2.734 \\
\hline
\end{tabular}

\section{Conclusions and future work}

In this paper, a novel long-term conflict avoidance ap-proach supporting the 4D-Trajectory (4DT) operation is proposed to provide better strategic flight flow manage-ment solutions. Taking the flights track 
error into considera-tion, the long-term conflict avoidance (LCA) problem is firstly formulated as a multiobjective problem minimizing the total delay and the number of conflicts simultaneously. Considering that the LCA problem is a large-scale combi-natorial optimization problem with tightly coupled varia-bles, in this work, a cooperative co-evolution (CC) algo-rithm is introduced to divide the complex problem into sev-eral low-dimensional sub-problems to further improve the searching capability. A dynamic grouping strategy based on the conflict between flights is proposed to improve the op-timization efficiency and avoid premature convergence. To fully utilize the proposed grouping strategy, the well-known multi-objective evolutionary algorithm based on decompo-sition (MOEA/D) is employed in search of better solutions for each sub-problems. The proposed approach has been validated using real traffic data from Chinese air route network, and the results demonstrate that the proposed approach obtained better non-dominated solutions than the existing approaches including the MOGA, NSGA2, and MOEA/D. The results also show that our approach can provide satisfactory solutions for controllers under real op-erational scenarios.

Acknowledgements This work was supported by the National Natural Science Foundation of China [Grant No. U1533119,and Grant No. U1433203], and the Foundation for In-novative Research Groups of the National Natural Science Foundation of China [Grant No. 61221061].

Conflict of interest The authors declare that they have no conflict of interest.

Supporting information Appendix A. The supporting information is available online at info.scichina.com and link.springer.com. The supporting materials are published as submitted, without typesetting or editing. The responsibility for scientific accuracy and content remains entirely with the authors.

\section{References}

1 Liu W, Hwang I. Probabilistic trajectory prediction and conflict detection for air traffic control. AIAA Journal of Guidance, Control and Dynamics, 2011, 34(6): 1779-1789.

2 Lv R L,Guan X M,Li X Y, et al. A large-scale flight multi-objective assignment approach based on multi-island parallel evolution algorithm with cooperative coevolutionary. Science China Information Sciences,2016,59(7):1-17.

3 Guan X M, Zhang X J, Han D, et al. A Strategic Flight Conflict Avoidance Approach based on Memetic Algo-rithm. Chinese Journal of Aeronautics, 2013, 27(1): 93C101.

4 Kuchar J, Yang L. A review of conflict detection and resolution modeling methods. IEEE Trans. Intell. Transp. Syst. 2000, 1(4): 179C189.

5 Hwang I, Tomlin C. Protocol-based conflict resolution for finite information horizon. In Proc. Amer. Control Conf., Anchorage, 2002, 748C753.

6 Archibald K., Hill J C, Jepsen N A, et al. A satisficing approach to aircraft conflict resolution. IEEE Trans. Syst., Man, Cybern. C, 2008, 38(4): 510C521.

7 Tomlin C, Mitchell I, Ghosh R. Safety verification of conflict resolution manoeuvres. IEEE Trans. Intell. Transp. Syst., 2001, 2(2): 110C120.

8 Kosecka K, Tomlin C, Pappas G., et al. Generation of con-flict resolution maneuvers for air traffic management. In Proc. IEEE/RSJ Int. Conf. Intell. Robots Syst. 1997, 1598C1603.

9 Mao Z H, Dugail D, Feron E. Space partition for conflict reso-lution of intersecting flows of mobile agents. IEEE Trans. Intell. Transp. Syst., 2007, 8(3): 512C527.

10 Pallottino L, Feron E M, Bicchi A. Conflict resolution problems for air traffic management systems solved with mixed integer programming. IEEE Trans. Intell. Transp. Syst. 2003, 3(1): 3C11.

11 Mondoloni S, Conway S. An airborne conflict resolution ap-proach using a genetic algorithm. Nat. Aeronautics Space Admin. Tech. Rep., 2001, AIAA-2001-4054.

12 Vivona R, Karr D, Roscoe D. Pattern based genetic algorithm for airborne conflict resolution. Presented at the AIAA Guid-ance, Navigation, Control Conf. Exhib, 2006, AIAA-2006-6060.

13 Pechoucek M, ?i?lk D. Agent-based approach to free-flight planning, control, and simulation. IEEE Intell. Syst., 2009, 24(1).

14 Hill J C, Johnson F R., Archibald J K., et al. A cooperative multi-agent approach to free flight. In Proc. 4th Int. Joint Conf. Auton.AgentsMultiagent Syst, 2005, 1083C1090.

15 Rong J, Geng S, Valasek J, et al. Air traffic control nego-tiation and resolution using an on board multi-aircraft system. In Proc. 21th Digital Avionics Syst. Conf., 2002, 7B2-1C7B2-12.

16 Durand N, Allignol, C. 4D-Trajectory deconfliction through de-par- ture time adjustment. Proc. ATM09,8th USA/Europe R\&D Seminar on Air Traffic Management. 2009

17 Durand N, Alliot J M., Noailles J. Automatic aircraft conflict resolution using genetic algorithms. Symposium on Applied Computing. 1996. 
18 Su J, Zhang X J, Guan X M. 4D-Trajectory Conflict Resolution Using Cooperative Coevolution. International Conference on Information Technology and Software Engineering, 2012, 387-395.

19 Yang Z, Tang K, Yao X. Large scale evolutionary optimization using cooperative co-evolution. Information Sciences, 2008, 178(15): 2985C299.

20 Ray T, Yao X. A cooperative coevolutionary algorithm with correlation based adaptive variable partitioning. In 845 Pro-ceedings of the Eleventh Congress on Evolutionary Computa-tion, 2009, 983C989.

21 Omidvar M N, Li X D, Yao X. Cooperative coevolution with delta grouping for large scale non-separable function optimiza-tion. In Proceedings of the IEEE Congress on Evolutionary Computation, 2010, 1762C1769.

22 Sayed E, Essam D, Sarker R A. Dependency identification tech-nique for large scale optimization problems. In Proceedings of the IEEE Congress on Evolutionary Computation, 2012, pp. 1 C8.

23 Potter M, Jong K D. A cooperative coevolutionary approach to function optimization. In: Proceedings of the Third Conference on Parallel Problem Solving from Nature, 1994, 249C257.

24 Zhang Q, Li H. MOEA/D: A multi-objective evolutionary algo-rithm based on decomposition. IEEE Trans on Evolutionary Computation, 2007, 11: 712C731.

25 Delahaye D, Sofiane O, Puechmorel S. Airspace congestion smoothing by multi-objective genetic algorithm. ACM Sympo-sium on Applied Computing, 2005, 907-912.

26 Deb K, Pratap A, Agarwal S. A fast and elitist multi-objective genetic algorithm: NSGA-II. IEEE Transactions on Evolution-ary Computing, 2002, 6(2): 182-197.

27 Prandini M, Hu J H, Lygeros J, et al. A Probabilistic Ap-proach to Aircraft Conflict Detection. IEEE Trans. Intell. Transp. Syst., 2000, 1(4), 199-220.

28 Zhang X J, Guan X M, Hwang I, et al. A Hybrid Distribut-ed-Centralized Conflict Resolution Approach for MultiAircraft based on Cooperative Co-evolutionary. Science China Infor-mation Sciences, 2013, 56(12): 1-16.

29 Zitzler E, Thiele L, Laumanns M. Performance assessment of multiobjective optimizers: An analysis and review. IEEE Trans on Evolutionary Computation, 2003, 7: 117-132.

30 Fleischer M. The measure of Pareto optima: Applications to multiobjective metaheuristics. Proceeding of the 2nd Int Conf on Evolutionary Multi-objective Optimization, 2003. 519-533.

31 Wilcoxon F. Individual comparisons by ranking methods. Bio-metrics Bulletin, 1945, 1: 80-83.

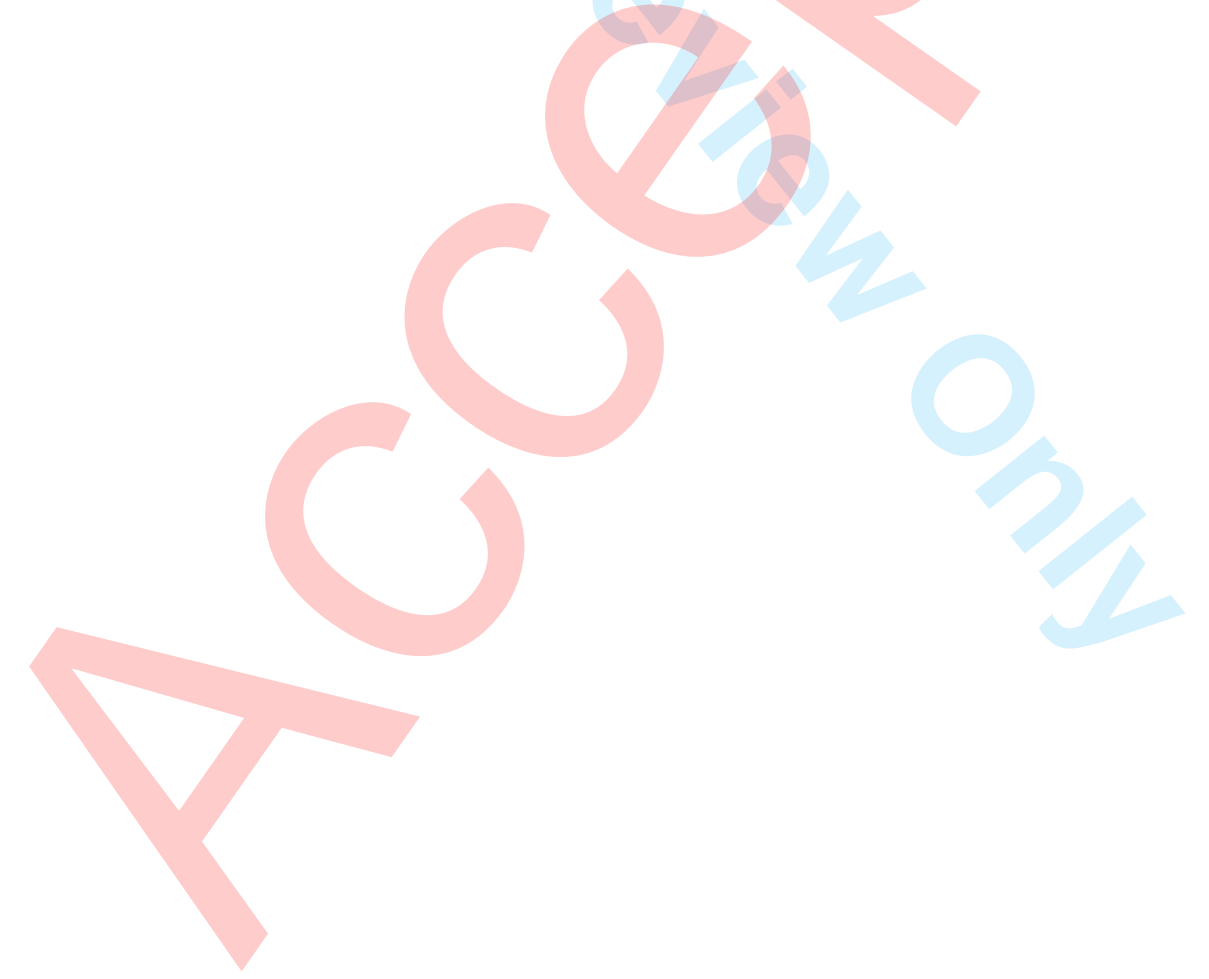

